\title{
Tighter Bounds on the Inefficiency Ratio of Stable Equilibria in Load Balancing Games
}

\author{
Akaki Mamageishvili and Paolo Penna \\ Department of Computer Science, ETH Zurich, Switzerland
}

November, 2015

\begin{abstract}
In this paper we study the inefficiency ratio of stable equilibria in load balancing games introduced by Asadpour and Saberi [3]. We prove tighter lower and upper bounds of $7 / 6$ and $4 / 3$, respectively. This improves over the best known bounds for the problem (19/18 and 3/2, respectively). Equivalently, the results apply to the question of how well the optimum for the $L_{2}$-norm can approximate the $L_{\infty}$-norm (makespan) in identical machines scheduling.
\end{abstract}

Keywords: Nash Equilibria; Load Balancing Games; Potential Games; Price of Anarchy.

\section{Introduction}

Load balancing problems are classical optimization problems which are also actively studied in the context of games, where jobs are owned by selfish but rational players. These games are prototypical of resource allocation problems in which users (players) do not act altruistically, therefore leading the system to suboptimal configurations. Naturally, one can consider the social optimum as the allocation minimizing the makespan, that is, the maximum load over all machines (a classical measure of efficiency). In contrast, in the game-theoretic setting, each player strives to optimize the cost of her own job only. This will typically result in a so-called Nash equilibrium, that is, an allocation in which no player can benefit by moving her job to another machine. In this work we consider pure Nash equilibria, that is, configurations in which each player chooses one strategy and unilateral deviations are not beneficial. In general, games may also possess mixed Nash equilibria in which players choose strategies according to a probability distribution. The inefficiency of Nash equilibria is a central topic in algorithmic game theory and it measures how much selfishness can impede optimization.

Asadpour and Saberi [3] introduced and studied the inefficiency ratio of stable equilibria (IRSE) in several games, including load balancing ones (see Section 2). This notion quantifies the efficiency loss in games when players play certain noisy best-response dynamics (see Section 1.2). For load balancing games, the IRSE has another very simple and natural interpretation (which is also of independent interest and studied earlier): 
Are the allocations minimizing the $L_{2}$-norm (sum of the squares of the machine loads) also sufficiently good for minimizing the $L_{\infty}$-norm (makespan)?

Intuitively, while the social cost is measured by the $L_{\infty}$-norm (makespan), the players collectively minimize the $L_{2}$-norm (the potential of the game). Therefore, the IRSE on load balancing games is equal to some value $c$ if every allocation minimizing the $L_{2}$-norm is automatically a $c$-approximation for the $L_{\infty}$-norm, i.e., the makespan of this allocation is at most $c$-times the optimal makespan. An exact bound on the IRSE is not known, as opposed to other measures related to the inefficiency of equilibria (see Section 1.2). Asadpour and Saberi [3] proved an upper bound of $3 / 2$ on IRSE and observed that an example in Alon et al. [1] implies a lower bound on IRSE of 19/18.

\subsection{Our contribution}

In this work we improve both the previous upper and lower bounds on the IRSE on load balancing games:

- In Section 3 we show an improved lower bound of $7 / 6$. While the previous lower bound from Alon et al. [1] is obtained by a simple instance with 3 machines and 6 jobs, our result is based on a family of instances that depend on the number of machines. Notably, our construction improves the previous lower bound already for three machines to 13/12. As the number of machines grows, the lower bound tends to $7 / 6$.

- In Section 4 we improve the upper bound to $4 / 3$. Intuitively speaking, the proof consists in showing that in every allocation whose cost is more than $4 / 3$ the optimal makespan, we can redistribute the jobs in a way that reduces the potential, i.e., the $L_{2}$-norm. This is the same argument of Asadpour and Saberi [3], and our technical contribution is to show that there are two ways to "reshuffle" the jobs, so that one of them decreases the potential.

As mentioned above, these results can be restated by saying that every job allocation minimizing the $L_{2}$-norm guarantees a $4 / 3$ approximation for the makespan, while in some instances the optimum for the $L_{2}$-norm has makespan at least $7 / 6$ the optimal makespan. Closing the gap between the upper and the lower bound remains an open question, which we discuss at the end of Section 4. In the next section, we discuss further relation with prior work, including studies on the quality of equilibria in games.

\subsection{Significance of the results and related work}

The inefficiency of Nash equilibria is often measured through two classical notions: the price of anarchy (PoA) introduced by Koutsoupias and Papadimitriou [9] for load balancing games on related machines, and the price of stability (PoS) introduced in Anshelevich et al. [2], which compare respectively the worst and the best Nash equilibrium to the social optimum. In some cases, these notions can be considered too extreme as they may include "unrealistic" equilibria.

The IRSE [3] studies the quality of equilibria selected by certain noisy best-response dynamics [4]. Intuitively, these dynamics will most likely rest on pure Nash equilibria 
minimizing the potential of the game, and the IRSE can be seen as the price of anarchy restricted to these selected equilibria. The IRSE is also known in the literature under the name of potential optimal PoA by Kawase and Makino [8], who also considered the analogous potential optimal PoS. Correa et al. [5] studied earlier potential optimal PoA in the capacitated network routing model, though under a different name. In load balancing games (and several others) we have $P o S<I R S E<P o A$, which in a sense tells that the PoA and the PoS are either too pessimistic or too optimistic. Specifically, on $m$ machines $P_{O A}=\left(2-\frac{1}{m+1}\right)$ [6, 10], $P o S=1$ [10], while IRSE is between $19 / 18$ and $3 / 2$ [3]. The latter bounds are strengthened in the present paper to $7 / 6 \leq I R S E \leq 4 / 3$. This means that players can easily compute a $4 / 3$ (or better) approximation of the optimum via these simple dynamics, but also that in some instances the dynamics is unlikely to choose optimal or nearly optimal solutions either (namely, within a factor smaller than $7 / 6)$.

The upper bound 4/3 also suggests an intriguing comparison with the study of sequential PoA by Hassin and Yovel [7] for these games: there, players are far from myopic and the equilibrium is meant on an extensive form game in which players are able to reason about future moves of following players. It is an interesting question which of these two dynamics give a better makespan in the end.

\section{Preliminaries}

In load balancing there are $n$ jobs with weights $w_{1}, \ldots, w_{n}$ that we want to put on $m$ identical machines (each job is allocated to one machine). The job allocation determines the load $l_{j}$ of each machine $j$, that is the sum of the jobs weights that are allocated to this machine. The goal is to find an allocation that has the lowest possible makespan, that is, the maximum load over all machines.

In load balancing games each job is a player who can choose any of the $m$ possible machines. The cost for player $i$ is simply the load of the machine chosen by this player, and naturally each player aims at minimizing her own cost. The strategies of all players specify a job allocation (in the game theoretic terminology this is the strategy profile).

An allocation minimizing the makespan is called a social optimum, and its makespan is called a social optimum makespan. The potential associated to an allocation is the sum of the squares of the corresponding machine loads, $l_{1}{ }^{2}+\cdots+l_{m}{ }^{2}$ where $l_{j}$ is the load of machine $j$ at this allocation. An allocation minimizing the potential function is called a potential optimal allocation or simply potential optimum.

It is well known that load balancing games are weighted potential games with the above potential function. This means that all pure Nash equilibria (allocations where no player can unilaterally improve moving to another machine) are actually 'locally optimal' for the potential (a single job move cannot reduce the potential). Asadpour and Saberi [3] introduced and studied the inefficiency ratio of stable equilibria (IRSE), which is the largest (among all the instances of a game) ratio between the worst makespan of a potential optimal allocation and the social optimum makespan.

Potential optimal allocations satisfy the following condition (see Figure 1). Split the total load of each machine into two bundles of jobs, that is, $l_{j}=x_{j}+y_{j}$ where $x_{j}$ is the sum of the weights of a (possibly empty) subset of jobs allocated to $j$. If two machines $i$ 


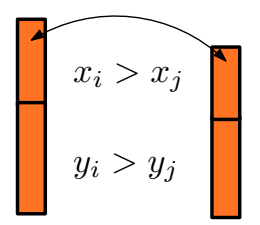

Figure 1: When a swap of bundles of jobs reduces the potential.
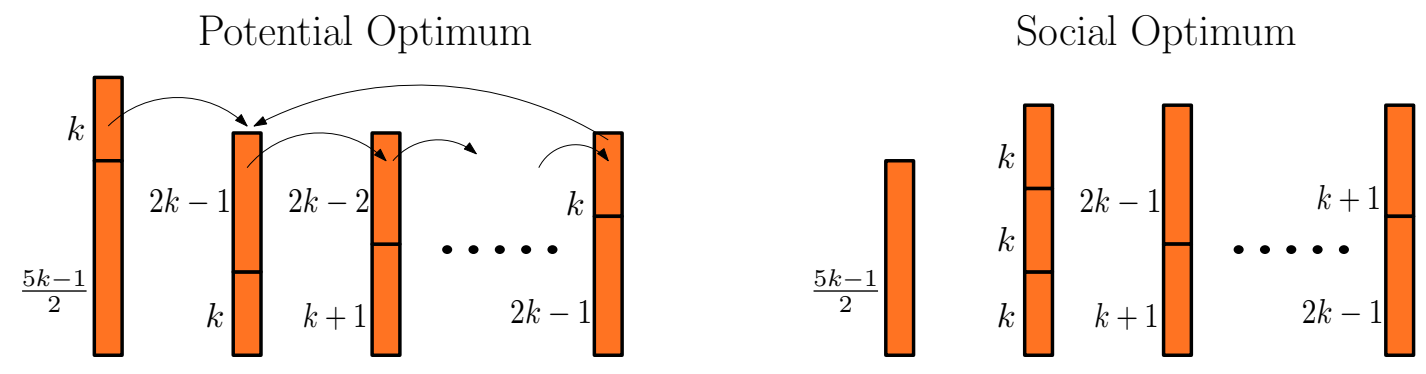

Figure 2: Lower bound $7 / 6$ on IRSE.

and $j$ satisfy $x_{i}<x_{j}$, then $y_{i} \geq y_{j}$ for otherwise swapping $x_{i}$ with $x_{j}$ reduces the potential. Pure Nash equilibria satisfy the weaker condition that a single job $k$ in machine $i$ does not improve if moving to another machine $j$, that is, $l_{i}-w_{k} \leq l_{j}$.

\section{Improved lower bound}

In this section we strengthen the 19/18 lower bound on IRSE in [3, 1]. The idea of the proof is to construct an instance in which the potential optimum is obtained when one single machine has "higher" load, and all others have the same load (Figure 2 left), while the optimal makespan does the opposite: one machine has "lower" load and all others have the same higher load (Figure 2 right).

Theorem 3.1. IRSE in load balancing games is at least $7 / 6$.

Proof. Consider the instance in Figure 2 where $m=k+1 \geq 3, n=2 k+2$ and the weights of jobs are $k, k, k, k+1, k+1, \ldots, 2 k-1,2 k-1,(5 k-1) / 2$. We prove that the allocation on the left (see figure) minimizes the potential function, while the one on the right has optimal makespan, thus implying

$$
I R S E \geq \frac{(7 k-1) / 2}{3 k}=\frac{7 m-8}{6 m-6}
$$

which tends to $7 / 6$ as $m$ goes to infinity. First note that the potential in both allocations is the same. Consider any job allocation and without loss of generality assume that the job with the largest weight is on machine 1. If we fix a load on machine 1, then any job allocation which balances the load on the other machines minimizes the potential (among all allocations with this fixed load on machine 1). Therefore, if the largest job is alone on machine 1 then the potential is minimized in the social optimum case, while if it is located on machine 1 together with the job of smallest weight then the potential is minimized when the job allocation is like on the left side of Figure 2. If the load on machine 1 is strictly larger than $(5 k-1) / 2+k$ then the allocation is not potential optimal, because of 


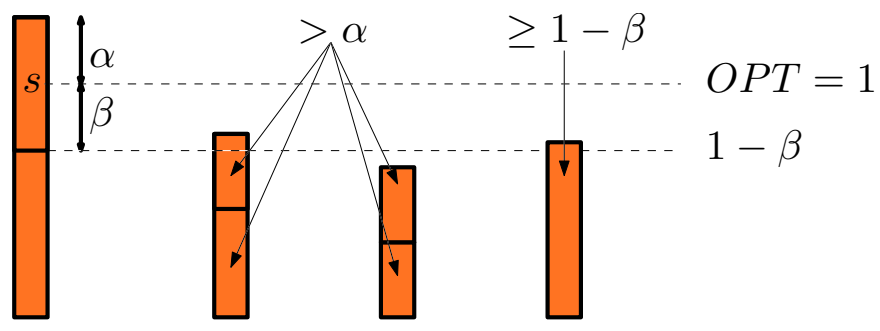

Figure 3: Proof of upper bound - Lemma 4.2.

property described in Figure 1. We conclude that both allocations are potential optimal, in particular the one on the left which gives the lower bound on IRSE.

\section{Improved upper bound}

Without loss of generality assume that a makespan in the social optimum is equal to 1 and the machine loads in the potential optimum are sorted in a non-increasing order $l_{1} \geq l_{2} \geq \cdots \geq l_{m}$.

Theorem 4.1. IRSE in load balancing games is at most $4 / 3$.

Proof. We have to prove that $l_{1} \leq 4 / 3$. Assume that it does not hold, that is $l_{1}=$ $1+\alpha>4 / 3$ implying $\alpha>1 / 3$. Machine 1 contains at least two jobs, otherwise the optimum makespan would not be 1 . The smallest job on machine 1 , which we call $s$, is strictly larger than $\alpha$, otherwise since $l_{m}<1$ we can decrease the potential by moving $s$ to the last machine. Assume that the weight of $s$ is $\alpha+\beta$ where $\beta>0$ and since $\alpha+\beta \leq l_{1}-\alpha-\beta=1-\beta$ it implies $\beta<1 / 3<\alpha$. The proof of the theorem is based on the following property of potential optimal allocations (whose proof is postponed).

Lemma 4.2. For any $\alpha>1 / 3$, in a potential optimal allocation, every machine must have one job of weight at least $1-\beta$ or two jobs of weight strictly bigger than $\alpha$.

Note that for $\alpha>1 / 3$ this contradicts the fact that the optimum makespan is equal 1 since any job from machine 1 has weight larger than $\alpha$ and together with these jobs cannot be allocated on $m-1$ machines without exceeding 1 (clearly the jobs in machine 1 cannot be together in the optimum makespan, no three jobs of weight $\alpha$ can fit together, nor a job of $1-\beta$ with a job of $\alpha$ ).

\subsection{Proof of Lemma 4.2}

We next prove Lemma 4.2. First observe that $l_{i} \geq 1-\beta$ for otherwise we can move job $s$ from machine 1 to machine $i$ and decrease the potential. Sort the jobs on machine $i$ in increasing weight, and consider a bundle of small jobs such that the load is still above or equal to $1-\beta$ after removing them from the machine (this bundle can be empty). Let $x$ be the overall weight of this bundle, and $y$ be the size of the next job whose removal reduces the load below $1-\beta$. That is

$$
l_{i}-x \geq 1-\beta \quad \text { and } \quad l_{i}-x-y<1-\beta .
$$



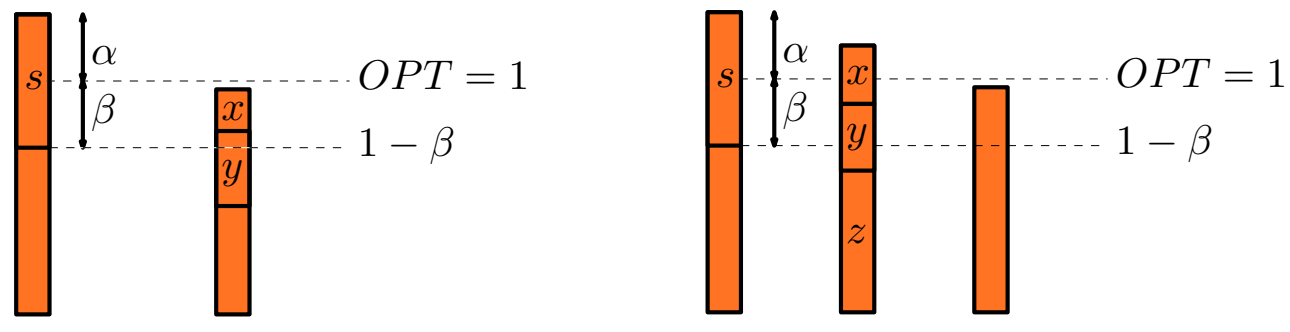

Figure 4: The two cases to prove Lemma 4.2.

Also note that

$$
x+y \geq \alpha+\beta
$$

otherwise we can exchange job $s$ on machine 1 with job bundle of weight $x+y$ on machine $i$ and strictly decrease the potential. If $y$ was the only job after removing $x$, then by definition $y \geq 1-\beta$ and the lemma holds. Otherwise, we show that $y \geq \alpha$. We next distinguish two cases depending on the load $l_{i}$ of machine $i$ (see Figure 4):

$\left(l_{i}<1\right)$. From the first inequality in (1) we get $x<\beta$, and then by (2) $y>\alpha$.

$\left(l_{i} \geq 1\right)$. If $x<\beta$ then $y>\alpha>1 / 3$ using the same argument of the previous case. Assume therefore $x \geq \beta$ and $y \leq 1 / 3<\alpha$. Let $z$ be the load of machine $i$ after removing the bundle of size $x+y$, that is, $l_{i}=x+y+z$. We rearrange the jobs in the potential optimum and show that this will further reduce the potential, thus a contradiction. The job rearrangement goes as follows:

1. Job $s$ moves from machine 1 to machine $i$;

2. The smallest between $x$ and $y$ moves to machine $m$, while the biggest moves to machine 1 .

Consider first the case $x \leq y$. Then the difference between potential of the original allocation and potential of the new one is

$$
\begin{gathered}
(1+\alpha)^{2}+(x+y+z)^{2}+l_{m}^{2}-(1-\beta+y)^{2}-(z+\alpha+\beta)^{2}-\left(l_{m}+x\right)^{2}= \\
2\left(\alpha+x y+y z+x z+\beta+\beta y-\left(\beta^{2}+y+\alpha z+\alpha \beta+z \beta+l_{m} x\right)\right)> \\
2\left(\alpha+x y+y z+x z+\beta+\beta y-\left(\beta^{2}+y+\alpha z+\alpha \beta+z \beta+x\right)\right)
\end{gathered}
$$

where the inequality is due to $l_{m}<1$. We next prove that this quantity is positive and obtain the desired contradiction. Note that $y+z \leq l_{m}$ otherwise moving the bundle of weight $x$ to machine $m$ reduces the potential. Therefore (3) is linear in $x$ with slope $y+z-1 \leq 0$ and since $x \leq y$, we can bound it from below by

$$
2\left(\alpha+y^{2}+2 y z+\beta+\beta y-\left(\beta^{2}+y+\alpha z+\alpha \beta+z \beta+y\right)\right) .
$$


This quantity is linear in $z$ with slope $2 y-(\alpha+\beta) \geq x+y-(\alpha+\beta) \geq 0$. Moreover, we have $z \geq 1-\beta-y$, since otherwise we could exchange job $s$ with bundle $y$ and decrease the potential. We can thus bound (4) from below by replacing $z$ with $1-\beta-y$ :

$$
\begin{array}{r}
2\left(\alpha+2 y-\beta y-y^{2}+\beta-\left(\beta^{2}+y+\alpha-\alpha y+\beta-\beta^{2}-\beta y+y\right)\right)= \\
2\left(y-\beta y-y^{2}+\beta-(-\alpha y+\beta-\beta y+y)\right)= \\
2\left(\alpha y-y^{2}\right)
\end{array}
$$

which is strictly positive since $y<\alpha$. This proves that (3) is strictly positive.

The case $y<x$ is similar to the previous one. Specifically, now the potential difference is strictly bigger than (3) but with $x$ and $y$ exchanged,

$$
2\left(\alpha+x y+y z+x z+\beta+\beta x-\left(\beta^{2}+x+\alpha z+\alpha \beta+z \beta+y\right)\right)
$$

and this quantity is linear in $y$ with non-positive slope $x+z-1 \leq 0$, since otherwise moving bundle $y$ to machine $m$ would reduce the potential. For the case $x<\alpha$ the rest of the proof is identical to the previous case with $x$ and $y$ exchanged. For $x \geq \alpha$ we use $y<\alpha$ and obtain a lower bound for (5) by replacing $y$ with $\alpha$, and then $z$ by $1-\beta-\alpha$ :

$$
\begin{array}{r}
2\left(\alpha+\alpha x+x z+\beta+\beta x-\left(\beta^{2}+x+\alpha \beta+z \beta+\alpha\right)\right)= \\
2\left(\alpha+\alpha x+x-x(\alpha+\beta)+\beta+\beta x-\left(\beta^{2}+x+\alpha \beta+\beta-\beta(\alpha+\beta)+\alpha\right)\right)=0
\end{array}
$$

where in the second step we use $x \geq \beta$ since $\beta<\alpha$ as observed before this lemma.

This completes the proof of Lemma 4.2.

Remark 4.3. We believe that our lower bound example can not be improved. In order to prove this claim one has to consider global properties of potential optimizer, while in our proof only local properties have been used. Specifically, in Lemma 4.2 we use a simple "reshuffling" of the jobs that involves only three bundles and three machines.

Acknowledgments. We wish to thank an anonymous reviewer for spotting a mistake in the proof of Lemma 4.2. This work has been partially supported by the Swiss National Science Foundation (SNF) under the grant number 200021_143323/1.

\section{References}

[1] Noga Alon, Yossi Azar, Gerhard J. Woeginger, and Tal Yadid. Approximation schemes for scheduling. In Proc. of the 8th Annual Symposium on Discrete Algorithms (SODA), pages 493-500, 1997.

[2] Elliot Anshelevich, Anirban Dasgupta, Jon M. Kleinberg, Éva Tardos, Tom Wexler, and Tim Roughgarden. The price of stability for network design with fair cost allocation. SIAM J. Comput., 38(4):1602-1623, 2008. 
[3] Arash Asadpour and Amin Saberi. On the Inefficiency Ratio of Stable Equilibria in Congestion Games. In Proc. of the 5th International Workshop on Internet and Network Economics (WINE), volume 5929 of LNCS, pages 545-552, 2009.

[4] Lawrence E. Blume. Population games. Addison-Wesley, 1998.

[5] José R. Correa, Andreas S. Schulz, and Nicolás E. Stier-Moses. Selfish routing in capacitated networks. Mathematics of Operations Research, 29(4):961-976, 2004.

[6] Ronald L. Graham. Bounds on multiprocessing timing anomalies. SIAM Journal of Applied Mathematics, 17(2):416-429, 1969.

[7] Refael Hassin and Uri Yovel. Sequential scheduling on identical machines. Oper. Res. Lett., 43(5):530-533, 2015.

[8] Yasushi Kawase and Kazuhisa Makino. Nash equilibria with minimum potential in undirected broadcast games. Theoretical Computer Science, 482:33-47, 2013.

[9] Elias Koutsoupias and Christos H. Papadimitriou. Worst-case equilibria. In Proc. of the 16th Annual Symposium on Theoretical Aspects of Computer Science (STACS), volume 1563 of $L N C S$, pages 404-413, 1999.

[10] Noam Nisan, Tim Roughgarden, Eva Tardos, and Vijay V. Vazirani. Algorithmic Game Theory. Cambridge University Press, New York, NY, USA, 2007. 